\title{
Retraction Note to: Genetic variations in the KIR gene family may contribute to susceptibility to ankylosing spondylitis: a meta-analysis
}

\author{
Hai-Ning Zuo ${ }^{1} \cdot$ Zhi-Long Wang $^{2} \cdot$ Dao-Ran $\mathrm{Cui}^{3} \cdot$ Da-Jiang Xin ${ }^{4}$
}

Published online: 18 August 2015

(C) Springer Science+Business Media Dordrecht 2015

Retraction Note to: Mol Biol Rep (2014) 41:5311-5319
DOI 10.1007/s11033-014-3402-4

The Publisher and Editor retract this article in accordance with the recommendations of the Committee on Publica tion Ethics (COPE). After a thorough investigation we have strong reason to believe that the peer review process was compromised.

The online version of the original article can be found under doi:10.1007/s11033-014-3402-4.

Da-Jiang Xin

xindajiang118@163.com

1 1st Department of Trauma Surgery, Yantai Hill Hospital of Yantai, Yantai 264000, People's Republic of China

2 Department of Hand Surgery, Yantai Hill Hospital of Yantai, Yantai 264000, People's Republic of China

3 Department of Orthopedics, The First People's Hospital of Nantong, Nantong 226000, People's Republic of China

4 3rd Department of Trauma Surgery, Yantai Hill Hospital of Yantai, Jiefang Road No. 91, Zhifu District, Yantai 264000, People's Republic of China 